\title{
The role of capsule endoscopy in etiological diagnosis and management of obscure gastrointestinal bleeding
}

\author{
Vikas Pandey, Meghraj Ingle, Nilesh Pandav, Pathik Parikh, Jignesh Patel, Aniruddha Phadke, \\ Prabha Sawant \\ Department of Gastroenterology, Lokmanya Tilak Municipal Medical College \& Lokmanya Tilak Municipal General Hospital, Mumbai, India
}

\begin{abstract}
Background/Aims: To investigate the various etiologies, yields, and effects of capsule endoscopy (CE) on management and complications, along with follow up of patients with obscure gastrointestinal (GI) bleeding. Methods: The study group of patients included those having obscure, overt, or occult GI bleeding. The findings were categorized as (A) obvious/definitive, (B) equivocal, or (C) negative. Any significant alteration in patient management post $\mathrm{CE}$ in the form of drug or surgical intervention was noted. Results: Total patients included in the study were 68 (48 males and 20 females). The ratio of male:female was 2.4:1. The age ranged between 16 years to 77 years. Mean age for males was $62 \pm 14$ years, for females $58 \pm 16$ years. The total yield of CE with definitive lesions was in 44/68 (65.0\%) of patients. In descending order (A) angiodysplasia 16/68 (23.53\%), (B) Crohn's disease 10/68 (14.70\%), (C) non-steroidal anti-inflammatory drug enteropathy 8/68 (11.76\%), (D) small bowel ulcers 4/68 (5.88\%), (E) jejunal and ileal polyps 2/68 (2.94\%), (F) intestinal lymphangiectasis 2/68 (2.94\%), and (G) ileal hemangiomas 2/68 (2.94\%) were followed. Equivocal findings 12/68 (17.65\%) and negative study 12/68 (17.65\%) was found. Complications in the form of capsule retention in the distal ileum were noted in 2/68 (2.94\%) subjects. Statistically, there was a higher probability of finding the etiology if the CE was done during an episode of bleeding. Conclusions: CE plays an important role in diagnosing etiologies of obscure GI bleeding. Its role in influencing the management outcome is vital. (Intest Res 2016;14:69-74)
\end{abstract}

Key Words: Etiology; Gastrointestinal bleeding; Capsule endoscopy; Angiodysplasia; Small bowel ulcer

\section{INTRODUCTION}

Obscure gastrointestinal (GI) bleeding (OGIB) is defined as GI bleeding of uncertain cause after a non-diagnostic upper GI endoscopy, colonoscopy, and barium meal follow through. OGIB can be occult or overt. Overt OGIB may have visible acute GI bleeding either melena or hematochezia and imaging modality. Occult OGIB refers to a positive fecal occult blood test, usually in association with iron deficiency anemia. Five percent of all cases of GI bleeding fall in the

Received June 15, 2015. Revised August 14, 2015.

Accepted August 22, 2015.

Correspondence to Vikas Pandey, Department of Gastroenterology,

Lokmanya Tilak Municipal Medical College \&t General Hospital, Sion, Mumbai

400022, India. Tel: +91-0224063000, Fax: +91-02224063033,

E-mail: reenaupadhyay66@gmail.com

Financial support: None. Conflict of interest: None. category of OGIB. In 3/4 th cases, the etiology resides in the small bowel. Modalities to visualize the small bowel are limited. Push enteroscopy visualizes $45-60 \mathrm{~cm}$ beyond the ligament of Trietz. ${ }^{1}$ Double balloon endoscopy visualizes $230 \mathrm{~cm}$ beyond the ligament of Trietz and $135 \mathrm{~cm}$ beyond, proximal to the ileo-cecal valve. ${ }^{2}$ Wireless Capsule Endoscopy (CE) visualizes the small bowel with good sensitivity and specificity in addition to it being non invasive and hence is much more acceptable. However, Indian data about its efficacy or diagnostic yield and ability to influence management of patients with OGIB are limited. If etiologies differ from those of the Western world, this needs to be evaluated. The study aimed at evaluating the causes of OGIB, the diagnostic yield of CE, the outcomes of these group of patients in terms of the rebleeding rates, and impact on the management.

\footnotetext{
๑ Copyright 2016. Korean Association for the Study of Intestinal Diseases. All rights reserved.

This is an Open Access article distributed under the terms of the Creative Commons Attribution Non-Commercial License (http://creativecommons.org/licenses/by-nc/4.0)

which permits unrestricted non-commercial use, distribution, and reproduction in any medium, provided the original work is properly cited.
} 


\section{METHODS}

\section{Study Design}

This is a prospective single center, observational study, carried out at Lokmanya Tilak Municipal General Hospital from December 2011 to December 2014. An informed consent was taken from all patients. The study had institutional Ethics Committee clearance (IRB No. IEC/25/13).

Patients, 18-80 years old, with OGIB were selected. Among them, (A) patients with ongoing, obscure overt GI bleeding, (B) patients with a history of GI bleeding in the last 15 days with negative upper endoscopy and ileocolonoscopy, (C) anemic patients with stool occult blood positive were included. Also, (A) patients with history of GI bleeding $>15$ days, (B) suspected intestinal stricture, (C) pregnancy, and (D) patients with intracardiac conducting devices were excluded in this study.

\section{Study Protocol}

The study was carried out by the GI department of Lokmanya Tilak Municipal Medical College and Sion Hospital Mumbai. The study group of patients were those having OGIB, who presented to our department or were referred to us. Before CE, thorough history, physical examination, and investigations, were reviewed. To avoid complications, all patients were subjected to either barium follow through or CT enteroclysis to rule out obvious stricture. The patients, prior to the day of capsule endoscopic examination, were

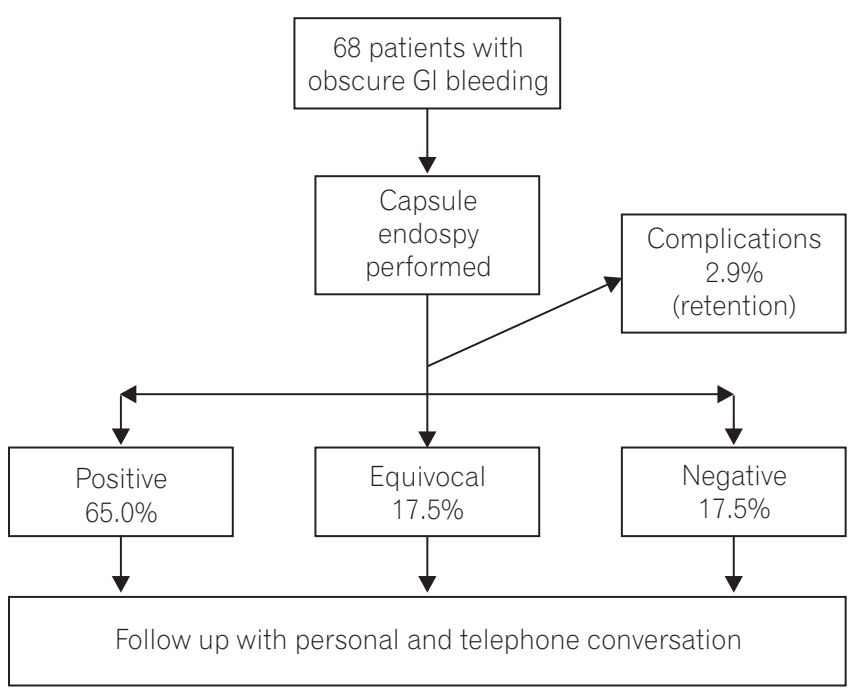

Fig. 1. Shows total number of patients enrolled, their outcomes and complication rates study design. $\mathrm{Gl}$, gastrointestinal. instructed to have their bowel prepared for endoscopy with polyethelene glycol. Patients were advised to fast on the day of examination. Patients swallowed the capsule with a glass of water. During the data recording, patients had the liberty to have light snacks after 4 hours. Total duration of the recording was approximately 8 hours. Patients were given an injectable prokinetic (metaclopromide) if capsule transit was slow (i.e., if the capsule remained in the stomach for more than 4 hours). Patients could be mobile if required (Fig. 1).

\section{Capsule endoscopy}

The procedure was carried out with $\mathrm{OMOM}^{\mathrm{TM}}$ (Chongqing Jinshan Science and Technology Company, Chongqing, China) capsule endoscopy (Fig. 2). It consisted of a lens, 4 light emitting diodes, a color camera, 2 batteries, a radiofrequency transmitter, and 14 antennas. Its length was $28 \mathrm{~mm}$ and weight was $6 \mathrm{gm}$. The battery half-life was 8 hours. The camera transmitted multiple (usually $2 / \mathrm{s}$ ) images by radiofrequency, through a sensor, to a recorder.

\section{Data Recording and Analysis}

Before patients swallowed the capsule, 8 skin electrodes were taped to the thorax and anterior abdominal wall. The capsule, while traversing the GI tract, captured images and sent these through radiofrequency transmitters. The sensor arrays, that were fixed at different locations on the anterior abdominal wall to the data logger, were attached to the patient. After study completion, the images were downloaded to a computer and seen as video images with software. The

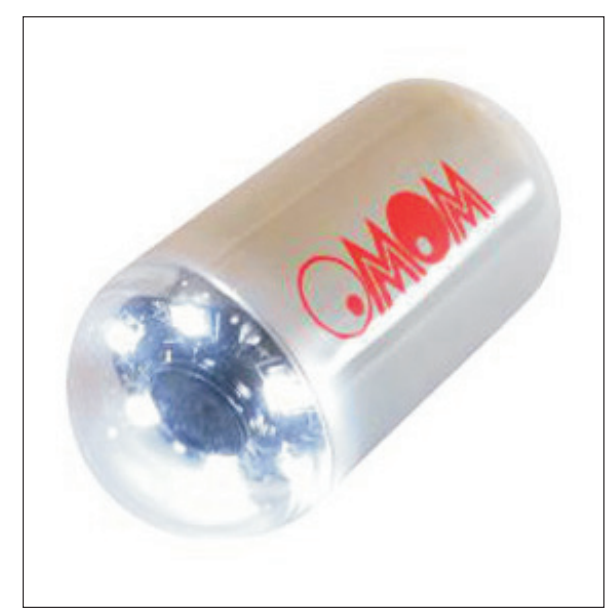

Fig. 2. $O M O M^{\mathrm{TM}}$ capsule endoscopy. 
images were interpreted by 2 experienced gastroenterologists, and the lesions were classified according to Saurin's classification ${ }^{3}$ into: $\mathrm{P} 0$ lesions that had no potential for bleeding, such as prominent submucosal veins, $\mathrm{P} 1$ lesions with uncertain hemorrhagic potential such as erosions, and P2 lesions, which had high potential for bleeding, such as angiodysplasias, varices, and large ulcers. The findings were categorized as (A) negative (P0) (B) obvious/definitive (P2), or (C) equivocal (P1). The study involved reporting all lesions from the stomach to the proximal colon. Any significant alteration in patient management post $\mathrm{CE}$, in the form of drug or surgical intervention, was noted. Follow up of patients were done as per outpatient department consultation or telephonic conversation.

\section{Statistical Analysis}

The numerical value results were expressed as a mean $\pm S D$. Chi square test was used for comparing of percentages. The $P$-value of $<0.005$ was considered significant. The SPSS 13.0 (SPSS Inc., Chicago, IL, USA) for windows was used for the statistical analysis.

\section{RESULTS}

\section{Findings}

Total patients included in the study were 68 (males 48, females 20). The ratio of male:female was 2.4:1. The ages ranged between 16 years to 77 years. Mean age for males was $62 \pm 14$ years, for females $58 \pm 16$ years. $32 / 68(47.05 \%)$ had active overt OGIB, 20/68 (29.5\%) had occult GI bleedings, and 16/68 (23.5\%) had a history of GI bleeding in last

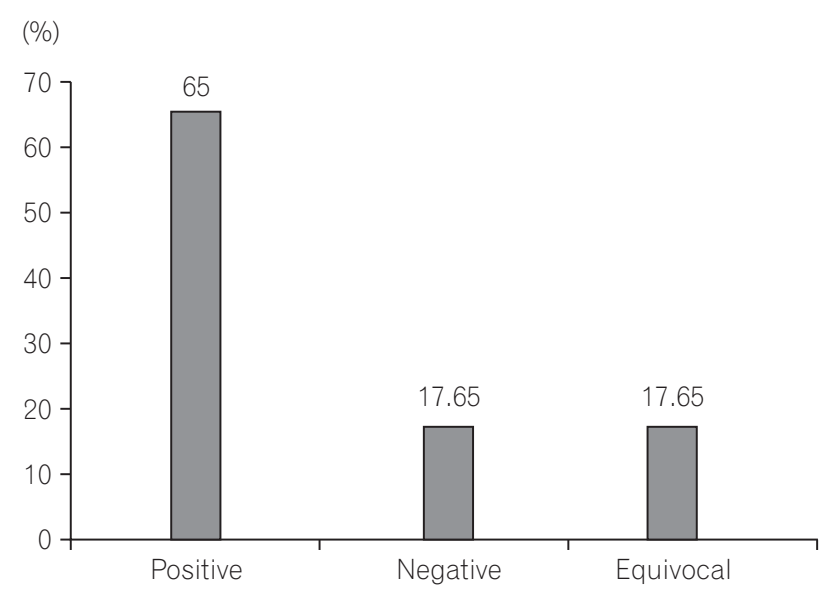

Fig. 3. Diagnostic yield of capsule endoscopy.
2 weeks. The total yield of CE with definitive P2 lesions was in 44/68 (65\%) of patients. Equivocal findings were seen in P1 12/68 (17.65\%). Negative study P0 was seen in 12/68 (17.65\%) (Fig. 3). In descending order, (Fig. 4) angiodysplasia 16/68 (23.53\%), CD 10/68 (14.7\%), NSAID enteropathy 8/68 (11.76\%), small bowel ulcers 4/68 (5.88\%), jejunal and ileal polyps $2 / 68$ (2.94\%), intestinal lymphangectasias $2 / 68$ (2.94\%), ileal hemangiomas 2/68 (2.94\%), jejunal varix 2/68 (2.94\%) (Fig. 5).

Complications in the form of capsule retention in the distal ileum was noted in 2/68 (2.94\%). Endoscopic insertion of the capsule was performed in 1/68 (1.47\%). On statistical analysis (Table 1), it was found that there was no difference between the type of bleeding (overt vs. occult) in finding a cause for the bleeding, but there was a higher probability of finding the etiology if the CE was done during an episode of active bleeding.

\section{Outcome}

\section{1) Rebleeding}

The rebleeding rate among those with positive findings was $9 / 44(20.45 \%)$, negative findings $1 / 12$ (8.3\%), and equivocal findings $0 / 12(0 \%)$. Among 9 rebleeding with positive findings, 4 had angiodysplasias, 3 had CD, and 1 had ileal hemangioma. One, with negative findings, who rebled, was diagnosed to have hemosuccus pancreaticus.

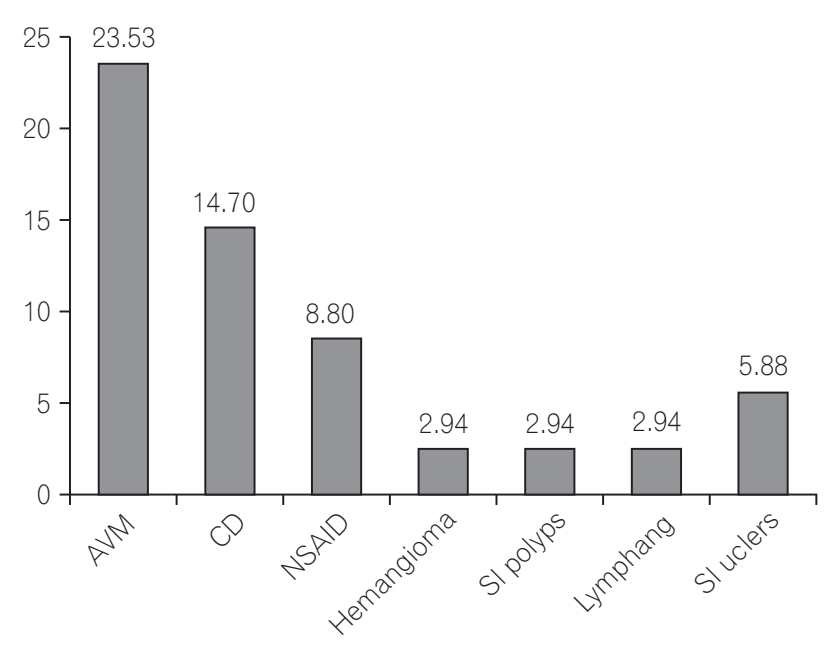

Fig. 4. Frequency of various etiologies observed in positive endoscopy findings. AVM, angiodysplasia; NSAID, NSAID enteropathy; SI, small intestinal; Lymphang, intestinal lymphangiectasias. 

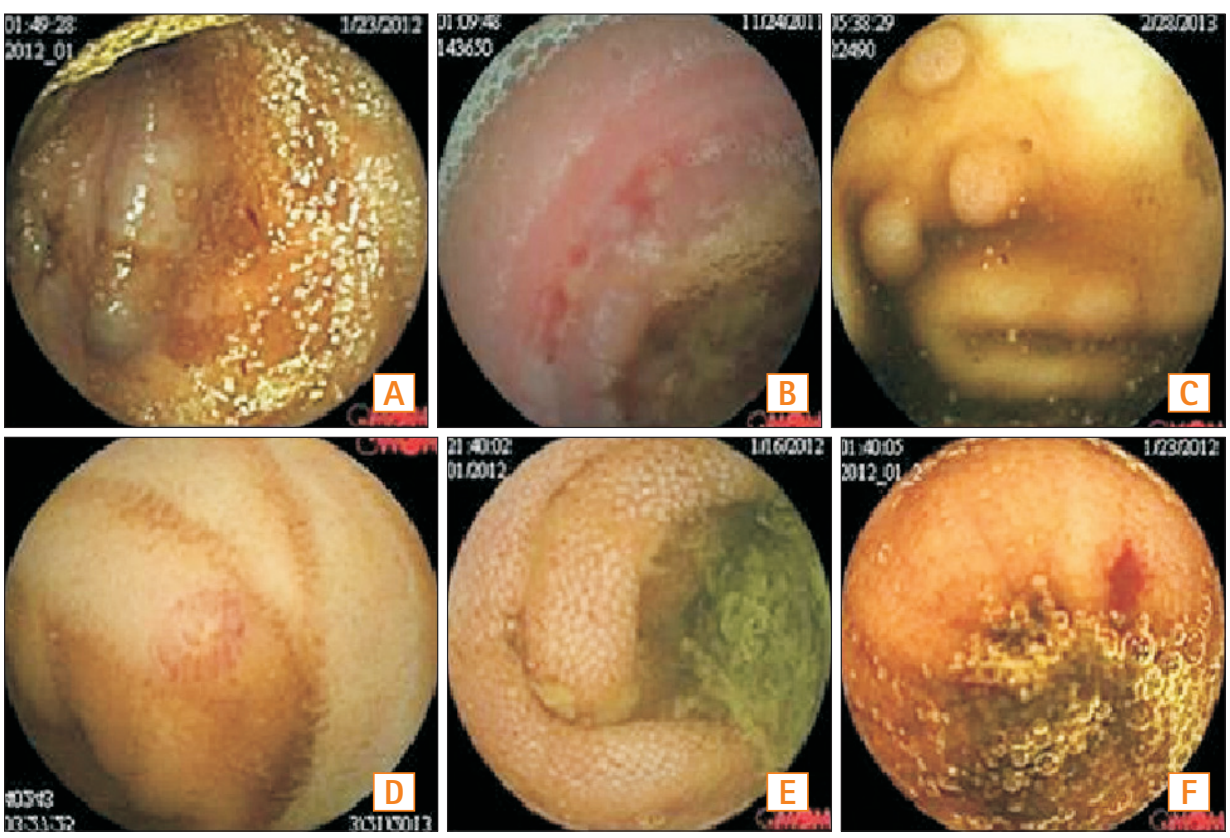

Fig. 5. (A) Jejunal varix, (B) CD, (C) Jejunal polyp, (D) Angiodysplasia, (E) Intestinal lymphangiectasias, (F) Small bowel ulcer (active bleeding).

Table 1. Data Analysis Depending on Type of Bleeding and Timing of Capsule Endoscopy

\begin{tabular}{lccccccc}
\hline \multirow{2}{*}{ Variable } & \multicolumn{3}{c}{ Type of bleeding } & & \multicolumn{3}{c}{ Time of capsule endoscopy } \\
\cline { 2 - 3 } & $\begin{array}{c}\text { Overt bleeding } \\
(\mathbf{n}=\mathbf{3 2})\end{array}$ & $\begin{array}{c}\text { Occult bleeding } \\
(\mathbf{n}=\mathbf{2 0})\end{array}$ & $\boldsymbol{P}$-value & & $\begin{array}{c}\text { Present bleeding } \\
(\mathbf{n}=\mathbf{5 2})\end{array}$ & $\begin{array}{c}\text { History of bleeding } \\
(\mathbf{n}=\mathbf{1 6})\end{array}$ & $P$-value \\
\hline Positive & 24 & 15 & 1.0000 & & 39 & 5 & 0.0013 \\
Equivocal/Negative & 8 & 5 & 1.0000 & 13 & 11 & 0.0013 \\
\hline
\end{tabular}

\section{2) Follow Up and Management}

In a follow up among 12 patients with negative study, one expired 6 months later with rupture of an abdominal aortic aneurysm, 3 were diagnosed with myelodysplastic syndrome, 2 hematological malignancy, 1 had a neuroendocrine tumor of duodenum, 1 was operated on for a gastro duodenal artery aneurysm (hemosuccus pancreaticus), 2 are still asymptomatic without any intervention, and 2 had been lost to follow up. Among those with positive findings, 4 patients with angiodysplasias also had an intermittent history of NSAID ingestion. Six of 16 patients with angiodysplasia in the proximal jejunum underwent argon plasma coagulation and did not report rebleeding, 4 reported rebleeding and required blood transfusions, rest 4 are on iron therapy, ethnylestradiol, and thalidomide, and 2 were lost to follow up.

All patients with CD were managed with steroids, salicylates, and azathioprine. One young male with CD, who had capsule retention, died 5 months later due perforative peritonitis. Patient with lymphangiectasias were on medium chain triglycerides diet and vitamin A, D, E, K supplementa- tion, and that with polyps did not undergo intervention and are asymptomatic. Two patients with jejunal varix and 1 with ileal hemangioma underwent bowel resection. Among 12 patients with equivocal results, 6 had an intermittent history of short duration NSAID ingestion.

\section{DISCUSSION}

In the present study, the diagnostic yield, etiology, and outcomes of 68 patients with OGIB who underwent CE in a single institution, were reported. Although there are no standard criteria for defining occult OGIB, for the purposes of this document, OGIB is designated as occult when there is no evidence of gross bleeding (e.g., unexplained iron deficiency anemia suspected to be caused by GI blood loss). ${ }^{4}$

Total yield of CE with definitive P2 lesions was obtained in $65 \%$ of patients. This is in accordance with diagnostic yield of CE published in a recent meta-analysis by Teshima et al. ${ }^{5}$ where the diagnostic yield was $62.7 \%$. The diagnostic yield reported in a previous Indian study was $52 \% .{ }^{6}$ One of the largest studies of Thailand had a low diagnostic yield of 
$36 \%{ }^{7}$ The low yield in this study was attributed to delay in performing $\mathrm{CE}$, which probably led to spontaneous healing of small bowel ulcers, which was the commonest finding in those patients. Various meta-analyses have reported a diagnostic yield of $61 \%-63 \%{ }^{8,9}$ The diagnostic yield was similar in both males and females.

Angiodysplasia (23\%) was the commonest etiology in our study. This is contrary to the Indian study ${ }^{6}$ and the Thailand study. ${ }^{7}$ The probable explanations to that are: First, the mean age of patients was comparatively older in the positive finding group, where angiodysplasia was the commonest finding, as compared to other groups. Zhang et al..$^{10}$ in their review had proposed angiodysplasia as the most common cause of OGIB in patients age $>65$ years. Second, patients with occult obscure bleeding and those with a history of GI bleeding in the past 15 days comprised a major group in our cohort. Among these groups, those who had positive findings for angiodysplasia, formed the major group as compared to those who presented with overt active bleeding. Koulaouzidise et al. ${ }^{11}$ in his systematic review, has affirmed to the above fact, where an analysis of multiple studies of CE in iron deficiency anemia yielded angiodysplasias as the commonest finding. However, the small sample size of our study could be the cofounding factor and needs to be validated by a larger cohort. The above two studies ${ }^{6,7}$ also had angiodysplasia as the second most common cause.

Patients with suspected CD on CE were confirmed histologically, either by antegrade or by retrograde enteroscopy, depending upon the location of the ulcer. One patient had surgical histopathological diagnosis, due to segmental terminal ileal resection, where the capsule became impacted. NSAID enteropathy was considered as the diagnosis in clinical context, when history of ongoing or recent i.e., in $<2$ weeks history of NSAID/aspirin consumption was available and histology was suggestive of inflammation. Those without this history were labeled as having had small bowel ulcers. We also observed intestinal lymphangectasia in 2 patients which was also seen in the previously published Indian data. ${ }^{6}$ Presentation of intestinal lymphangectasia associated with stool occult blood has been described by Freeman and Nimmo. ${ }^{12}$ We did not have small bowel tumor as a finding, which was seen in other studies. ${ }^{7}$ This was similar to other Indian studies. ${ }^{6}$ This probably could be explained by overall low prevalence of the disease. ${ }^{13}$ Reports of parasitic infections, identified through CE, have been described through case reports. ${ }^{14}$ However, no such findings were observed in our study. Multiple polyps in the entire small bowel were seen in 2 patients, which was not a common finding in other studies.

Rebleeding rate was highest in the group with positive findings (P2) lesion and least in the group with equivocal (P1) findings. This differs from the Thailand study ${ }^{7}$ where those with negative findings had the highest rebleeding rates. The possible explanation to that would be the higher rates of rebleeding in patients with angiodysplasia (commonest finding) which were inaccessible to argon plasma anticoagulation and were widespread throughout the small bowel. Capsule retention was the only complication noted in 2 patients. Endoscopic removal of the capsule was done from ileum who had NSAID enteropathy. In another patient, surgical removal was required, and whose postoperative specimen was suggestive of CD. Interestingly, pre-procedural CT eneteroclysis in both patients were normal.

Management has been influenced by CE findings in the form of argon plasma coagulation, salicylates, steroids and/ or azathioprine, surgical resection, and discontinuation of NSAID, were employed, depending upon the specific etiologies. Patients with negative/equivocal findings can be safely followed up due to lower rebleeding rates. One of the findings was that there was no difference between the type of bleeding (overt vs. occult) in finding a cause for the bleeding. This was in contradiction to the earlier studies published. Pennazio et al. ${ }^{15}$, reported that the highest video capsule endoscopy (CE) yield was in patients with active bleeding $(92.3 \%)$ or occult bleeding (44.2\%), whereas patients with previous overt bleeding had the lowest yield (12.9\%). The probable explanation to that is in both these group of patients, the CE was performed during the period of active bleeding, which could have led to lack of achieving statistical significance.

The other conclusion was that there is a higher probability of finding the etiology if the CE is done during an active episode of bleeding. Katsinelos et al. ${ }^{16}$ evaluated whether the timing influences diagnostic yield of CE. In their study, the diagnostic yield was 14/16 (87.5\%) in patients with overt bleeding, in those who underwent CE during the first 10 days following the bleeding episode, while it was only $1 / 9$ (11.1\%) for those who had CE more than 10 days afterwards. Singh et al. ${ }^{17}$ suggested that CE, if performed within 3 days of hospitalization, leads to higher diagnostic yields, higher therapeutic intervention rates, and decreased hospital stays. Therefore, the dictum should be performing CE during the period of active bleeding, as delayed study probably leads to spontaneous resolution of the findings, which could change the course of management of the condition. If we perform CE during active bleeding, there is a statistically higher 
chance of getting a positive result. Similarly, if there is history of bleeding in the past, there is a statistically higher chance of getting a negative result as compared to those with bleeding during CE. This implies that we should perform the CE during the bleeding itself, rather than utilizing it as a last resort. The later the CE is done, the less the likelihood of a positive result. Those with negative or equivocal findings can be safely followed up, due to lower rebleeding rates in these subgroups.

The study is highlighted by its prospective design and interpretation of CE findings by experienced gastroenterologists. However, the drawbacks being the low sample size and lack of adequate long follow up period were limitations. The results need to be validated by a larger cohort.

\section{REFERENCES}

1. Sidhu R, McAlindon ME, Kapur K, Hurlstone DP, Wheeldon MC, Sanders DS. Push enteroscopy in the era of capsule endoscopy. J Clin Gastroenterol 2008;42:54-58.

2. May A, Nachbar L, Schneider M, Ell C. Prospective comparison of push enteroscopy and push-and-pull enteroscopy in patients with suspected small-bowel bleeding. Am J Gastroenterol 2006;101:2016-2024.

3. Saurin JC, Delvaux M, Gaudin JL, et al. Diagnostic value of endoscopic capsule in patients with obscure digestive bleeding: blinded comparison with video push-enteroscopy. Endoscopy 2003;35:576-584.

4. ASGE Standards of Practice Committee; Fisher L, Lee Krinsky $\mathrm{M}$, et al. The role of endoscopy in the management of obscure GI bleeding. Gastrointest Endosc 2010;72:471-479.

5. Teshima CW, Kuipers EJ, van Zanten SV, Mensink PB. Double balloon enteroscopy and capsule endoscopy for obscure gastrointestinal bleeding: an updated meta-analysis. J Gastroenterol Hepatol 2011;26:796-801.

6. Gupta R, Lakhtakia S, Tandan M, et al. Capsule endoscopy in obscure gastrointestinal bleeding--an Indian experience. Indian J Gastroenterol 2006;25:188-190.
7. Pongprasobchai S, Chitsaeng S, Tanwandee T, Manatsathit S, Kachintorn U. Yield, etiologies and outcomes of capsule endoscopy in Thai patients with obscure gastrointestinal bleeding. World J Gastrointest Endosc 2013;5:122-127.

8. Triester SL, Leighton JA, Leontiadis GI, et al. A meta-analysis of the yield of capsule endoscopy compared to other diagnostic modalities in patients with obscure gastrointestinal bleeding. Am J Gastroenterol 2005;100:2407-2418.

9. Liao Z, Gao R, Xu C, Li ZS. Indications and detection, completion, and retention rates of small-bowel capsule endoscopy: a systematic review. Gastrointest Endosc 2010;71:280-286.

10. Zhang BL, Chen CX, Li YM. Capsule endoscopy examination identifies different leading causes of obscure gastrointestinal bleeding in patients of different ages. Turk J Gastroenterol 2012;23:220-225.

11. Koulaouzidis A, Rondonotti E, Giannakou A, Plevris JN. Diagnostic yield of small-bowel capsule endoscopy in patients with iron-deficiency anemia: a systematic review. Gastrointest Endosc 2012;76:983-992.

12. Freeman HJ, Nimmo M. Intestinal lymphangiectasia in adults. World J Gastrointest Oncol 2011;3:19-23.

13. Mohandas KM, Desai DC. Epidemiology of digestive tract cancers in India. V. Large and small bowel. Indian J Gastroenterol 1999;18:118-121.

14. Rao C, Sharma A, Rana SS, et al. Massive obscure overt gastrointestinal bleed: an unusual cause diagnosed by capsule endoscopy. J Postgrad Med Edu Res 2012;46:37-39.

15. Pennazio M, Santucci R, Rondonotti E, et al. Outcome of patients with obscure gastrointestinal bleeding after capsule endoscopy: report of 100 consecutive cases. Gastroenterology 2004;126:643-653.

16. Katsinelos P, Chatzimavroudis G, Terzoudis S, et al. Diagnostic yield and clinical impact of capsule endoscopy in obscure gastrointestinal bleeding during routine clinical practice: a singlecenter experience. Med Princ Pract 2011;20:60-65.

17. Singh A, Marshall C, Chaudhuri B, et al. Timing of video capsule endoscopy relative to overt obscure GI bleeding: implications from a retrospective study. Gastrointest Endosc 2013;77:761766. 